\section{HIGHLY MULTIPLEXED DIGITAL SPATIAL PROFILING OF THE TUMOR MICROENVIRONMENT OF NON-SMALL- CELL LUNG CANCER (NSCLC)}

${ }^{1}$ Arutha Kulasinghe*, ${ }^{2}$ Connor O'Leary, ${ }^{2}$ Rahul Ladwa, ${ }^{3}$ Majid Warkiani, ${ }^{1}$ Kenneth O'byrne. ${ }^{1}$ Queensland University of Technology, Kelvin Grove, Australia; ${ }^{2}$ Princess Alexandra Hospital, Woolloongabba, Australia; ' University of Technology Sydney, Sydney, Australia

Background Profiling the tumour microenvironment (TME) has been informative in understanding the underlying tumourimmune interactions. Multiplex immunohistochemistry(mIHC) coupled with molecular barcoding technologies have revealed greater insights into the TME.

Methods In this study, we utilised the Nanostring GeoMX Digital Spatial Profiler (DSP) platform to profile a NSCLC tissue microarray for protein markers across immune cell profiling, immuno-oncology(IO) drug target, immune activation status, immune cell typing, and pan-tumour protein modules. Regions of interest (ROIs) were selected that described tumour, TME and normal adjacent tissue (NAT) compartments.

Results Our data revealed that paired analysis $(n=18)$ of patient matched compartments indicated that the TME was significantly enriched in CD27, CD3, CD4, CD44, CD45, CD45RO, CD68, CD163, and VISTA relative to tumour. Unmatched analysis indicated that the $\operatorname{NAT}(n=19)$ was significantly enriched in CD34, fibronectin, IDO1, LAG3, ARG1 and PTEN when compared to the TM E $(n=32)$. Univariate Cox proportional hazards indicated that the presence of cells expressing CD3 (HR:0.5, p=0.018), CD34(HR:0.53, $\mathrm{p}=0.004)$ and ICOS (HR:0.6, p=0.047) in tumour compartments were significantly associated with improved overall survival (OS).

Conclusions We implemented both high-plex and highthroughput methodologies to the discovery of protein biomarkers and molecular phenotypes within biopsy samples and demonstrate the power of such tools for a new generation of pathology research.

Acknowledgements This study was funded by the Princess Alexandra Hospital Foundation grant for KOB. AK is supported by an NHMRC ECF Fellowship (APP1157741) and Cure Cancer (APP1182179).

Ethics Approval The study was approved by the QUT Human Research Ethics Board

http://dx.doi.org/10.1136/jitc-2020-SITC2020.0043

\section{$44 \quad$ BODY COMPOSITION MAY BE PROGNOSTIC AND PREDICTIVE OF CLINICAL OUTCOMES IN METASTATIC RENAL CELL CARCINOMA (MRCC) PATIENTS TREATED WITH IMMUNE CHECKPOINT INHIBITORS (ICI)}

${ }^{1}$ Dylan Martini* ${ }^{1}$ 'T Anders Olsen, ${ }^{2}$ Subir Goyal, ${ }^{2}$ Yuan Liu, ${ }^{1}$ Sean Evans, ${ }^{1}$ Benjamin Magod, 'Jacqueline Brown, ${ }^{3}$ Lauren Yantorni, ${ }^{3}$ Greta Russler, ${ }^{1}$ Sarah Caulfield, 'Jamie Goldman, 'Bassel Nazha, 'Wayne Harris, 'OMer Kucuk, 'Bradley Carthon, 'Viraj Master, ${ }^{1}$ Mehmet Bilen. ${ }^{1}$ Emory University School of Medicine, Atlanta, GA, USA; ${ }^{2}$ Emory University, Atlanta, GA, USA; ${ }^{3}$ Winship Cancer Institute, Atlanta, GA, USA

Background Immune checkpoint inhibitors (ICI) have revolutionized the treatment of metastatic renal cell carcinoma (mRCC). Biomarkers for mRCC patients treated with ICI are limited, and body composition is underutilized in mRCC. We investigated the association between body composition and clinical outcomes in ICI-treated mRCC patients.

Methods We performed a retrospective analysis of 79 ICItreated mRCC patients at Winship Cancer Institute from
2015-2020. Patients with CT scans within 2 months of ICIinitiation were included. Baseline CT images were collected at mid-L3 and segmented using SliceOMatic v5.0 (TomoVision) Density of skeletal muscle (SM), subcutaneous fat, inter-muscular fat, and visceral fat were measured and converted to indices by dividing by height $(\mathrm{m}) 2$ (SMI, SFI, IFI, and VFI, respectively). Total fat index (TFI) was defined as the sum of SFI, IFI, and VFI. Patients were characterized as high versus low for each variable at gender-specific optimal cuts using overall survival (OS) as the primary outcome. A prognostic risk score was created based on the beta coefficient from the multivariable Cox model (MVA) after best subset variable selection. Body composition risk score was calculated as IFI + $2 * S M$ mean + SFI, and patients were classified as high (0-1), intermediate (2), or low-risk (3-4). Kaplan-Meier method and Log-rank test were used to estimate OS and PFS and compare the risk groups. Concordance statistics (C-statistics) were used to measure the discriminatory magnitude of the model.

Results Most were male (73\%), and median age was 61 years. Patients were primarily intermediate $(54 \%)$ or poor-risk $(30 \%)$ per IMDC criteria and most received ICI as first $(35 \%)$ or second-line $(51 \%)$ therapy. The body composition high-risk patients had significantly shorter OS (HR: 6.37, p<0.001), PFS (HR: 4.19, $\mathrm{p}<0.001$ ), and lower chance of CB (OR: $0.23, \mathrm{p}=0.044$ ) compared to low-risk patients in MVA (table 1). Patients with low TFI had significantly shorter OS (HR: 2.72, $\mathrm{p}=0.002$ ), PFS (HR: 1.91, $\mathrm{p}=0.025$ ), and lower chance of CB (OR: $0.25, p=0.008)$ compared to high TFI patients in MVA. The C-statistics were higher for body composition risk groups and TFI compared to IMDC and BMI (table 2). The median OS and PFS were shorter for high-risk versus intermediate and low-risk patients (figures 1-2).

Abstract 44 Table 1 MVA* of association between body composition risk groups and TFI with clinical outcomes

\begin{tabular}{|c|c|c|c|c|c|c|}
\hline & \multicolumn{2}{|l|}{ OS } & \multicolumn{2}{|c|}{ PFS } & \multicolumn{2}{|c|}{$\mathbf{C B}$} \\
\hline & HR (CI) & p-value & HR (CI) & p-value & OR (CI) & p-value \\
\hline \multicolumn{7}{|c|}{ Body Composition Risk Group Analysis } \\
\hline \multirow[t]{2}{*}{$\begin{array}{l}\text { High Risk: } \\
\text { Risk Score }=0-1 \\
n=20\end{array}$} & \begin{tabular}{|l|}
6.37 \\
$(2.40-$ \\
$16.92)$ \\
\end{tabular} & $<0.001^{* *}$ & \begin{tabular}{|l|}
4.19 \\
$(1.87-$ \\
$9.42)$ \\
\end{tabular} & $<0.001^{* *}$ & \begin{tabular}{|l|}
0.23 \\
$(0.05-$ \\
$0.96)$ \\
\end{tabular} & $0.044^{\star *}$ \\
\hline & \multicolumn{2}{|c|}{\begin{tabular}{|l|} 
Median Survival: \\
6.3 months \\
\\
24-Month Survival: \\
$29.2 \%$
\end{tabular}} & \multicolumn{2}{|c|}{\begin{tabular}{|l|} 
Median Survival: \\
2.5 months \\
\\
12-Month Survival: \\
$15.0 \%$
\end{tabular}} & & \\
\hline \multirow[t]{2}{*}{\begin{tabular}{|l|} 
Intermediate \\
Risk: \\
Risk Score $=2$ \\
$n=42$ \\
\end{tabular}} & \begin{tabular}{|l|}
1.56 \\
$(0.61-$ \\
$3.95)$ \\
\end{tabular} & 0.350 & \begin{tabular}{|l|}
2.05 \\
$(0.98-$ \\
$4.29)$ \\
\end{tabular} & 0.057 & \begin{tabular}{|l|}
0.49 \\
$(0.15-$ \\
$1.59)$
\end{tabular} & 0.238 \\
\hline & \multicolumn{2}{|c|}{\begin{tabular}{|l|} 
Median Survival: \\
24.6 months \\
\\
24-Month Survival: \\
$53.1 \%$
\end{tabular}} & \multicolumn{2}{|c|}{$\begin{array}{l}\text { Median Survival: } \\
4.8 \text { months } \\
\\
\text { 12-Month Survival: } \\
26.6 \%\end{array}$} & & \\
\hline \multirow[t]{2}{*}{$\begin{array}{l}\text { Low Risk: } \\
\text { Risk Score }=3-4 \\
n=18 \\
\end{array}$} & 1 & & 1 & & 1 & \\
\hline & \multicolumn{2}{|c|}{\begin{tabular}{|l|} 
Median Survival: \\
44.5 months \\
\\
24-Month Survival: \\
$82.1 \%$
\end{tabular}} & \multicolumn{2}{|c|}{\begin{tabular}{|l|} 
Median Survival: \\
12.4 months \\
\\
12-Month Survival: \\
$54.3 \%$
\end{tabular}} & & \\
\hline \multicolumn{7}{|c|}{ Categorical Total Fat Index (TFI) Analysis*** } \\
\hline $\begin{array}{l}\text { Low } \\
n=34\end{array}$ & \begin{tabular}{|l|}
2.72 \\
$(1.43-$ \\
$5.17)$ \\
\end{tabular} & $0.002^{* *}$ & \begin{tabular}{|l|}
1.91 \\
$(1.09-$ \\
$3.35)$ \\
\end{tabular} & $0.025^{* *}$ & \begin{tabular}{|l|}
0.25 \\
$(0.09-$ \\
$0.70)$
\end{tabular} & $0.008^{* *}$ \\
\hline $\begin{array}{l}\text { High } \\
\mathrm{n}=45\end{array}$ & 1 & & 1 & & 1 & \\
\hline
\end{tabular}

